\title{
Primary Upper Urinary Tract Small Cell Carcinoma: A Case Series and Literature Review
}

\author{
Patrick J. Hensley, MD, Amul A. Bhalodi, MD, and Shubham Gupta, MD
}

\begin{abstract}
Background: Primary upper urinary tract small cell carcinoma (SCC) is exceedingly rare with $<30$ cases reported in the literature. Little is known about the incidence, diagnosis, treatment, and outcomes in these patients. We present a series of three patients with primary upper tract SCC.

Case Presentation: Patient 1 is an 89-year-old Caucasian male who presented with hydroureteronephrosis and a mass in the proximal right ureter. Biopsy revealed SCC. Without further intervention, the patient died 2 months after his diagnosis. Patient 2 is a 67-year-old Caucasian female who underwent left laparoscopic nephroureterectomy for primary distal ureteral SCC, pT4N1M0. She developed lymphadenopathy and completed external beam radiation to the pelvis and four courses of cisplatin-based chemotherapy. She died from metastatic disease 7 months after diagnosis. Patient 3 is a 45-year-old female who underwent open right radical nephrectomy, retroperitoneal lymph node dissection, and hepatic metastasectomy for metastatic primary upper tract SCC, pT3N1M1. She underwent two subsequent retroperitoneal debulking procedures for recurrence followed by treatment with octreotide. She developed widespread metastasis and was treated with temozolomide and capecitabine before her death 80 months after diagnosis.

Conclusion: This series contributes to the limited knowledge of the management and natural course of primary upper tract SCC. Patient 1 represents the first disease-specific mortality reported in a patient who received no therapy. Patient 3 represents the longest reported survival with metastatic disease, and the first treated with octreotide. The patient was managed with aggressive repeat surgical resection and exhibited 2 years of progression-free survival on octreotide. Emphasis should be placed on aggressive resection of all visible disease combined with the use of multimodal adjuvant chemoradiation for selected patients in this rare disease.
\end{abstract}

Keywords: ureter, upper tract, small cell carcinoma, neuroendocrine carcinoma, Goblet Sign

\section{Introduction and Background}

$\mathbf{P}$ RIMARY SMALl CELl CARCINOMA (SCC) of the genitourinary tract is a rare entity, comprising an estimated $0.7 \%$ of all bladder malignancies. ${ }^{1}$ Primary upper tract SCC is exceedingly rare, with literature limited to $<30$ case reports. $^{2-4}$ We report a series of three cases of primary SCC of the upper urinary tract, with discussion of clinical presentation, therapeutic course, and long-term outcomes.

\section{Case 1}

An 89-year-old Caucasian male presented with right flank pain and gross hematuria with clot retention. He had a remote history of smoking pipe tobacco. Medical history included coronary artery disease with coronary stents. His Eastern Cooperative Oncology Group (ECOG) performance status was 2. The patient had acute renal insufficiency with serum creatinine of $2.03 \mathrm{mg} / \mathrm{dL}$ (baseline $<1 \mathrm{mg} / \mathrm{dL}$ ).

A noncontrasted CT of the abdomen and pelvis showed moderate right hydroureteronephrosis secondary to a mass in the proximal right ureter (Fig. 1A). The patient underwent cystoscopy, bilateral retrograde pyelography, and right ureteroscopy. A classic "Goblet Sign" was identified on retrograde pyelography (Fig. 2A) and ureteroscopy revealed a large, friable, endophytic mass obliterating the lumen of the proximal ureter. Cold cup biopsies were obtained through a semirigid ureteroscope and a ureteral stent was placed.

Department of Urology, University of Kentucky College of Medicine, Lexington, Kentucky.

(C) Patrick J. Hensley et al. 2017; Published by Mary Ann Liebert, Inc. This is an Open Access article distributed under the terms of the Creative Commons Attribution License, which permits unrestricted use, distribution, and reproduction in any medium, provided the original work is properly cited. 

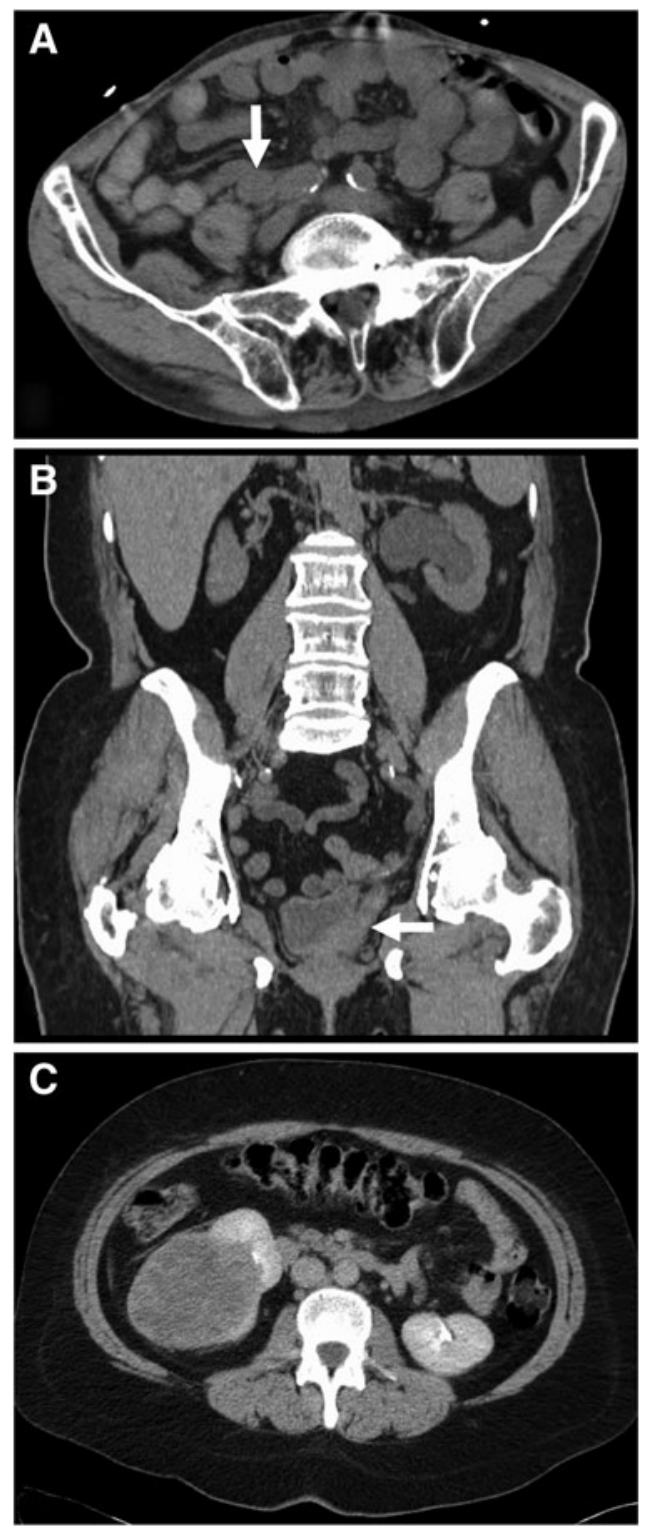

FIG. 1. Axial noncontrast CT section from Case 1 depicting a proximal right ureteral mass (white arrow, A). Coronal noncontrast CT section from Case 2 depicting a distal left ureteral mass (white arrow, B). Axial section of excretory phase-contrasted CT from Case 3 depicting a large, heterogeneous, enhancing right renal mass $(\mathbf{C})$.

Histologic evaluation revealed SCC characterized by positive immunohistochemical staining for CAM 5.2 (Fig. 2B, C).

After consultation with medical oncology, the patient opted for surveillance of the mass secondary to his age and deconditioning. He was admitted to a hospice service and died 2 months after diagnosis.

\section{Case 2}

A 67-year-old Caucasian female presented with gross hematuria and left flank pain. She denied history of tobacco use and medical history included obesity, hypertension, hyperlipidemia, anxiety, depression, and asthma. Her ECOG performance status was 1 . Imaging showed a distal left ureteral mass (Fig. 1B) and
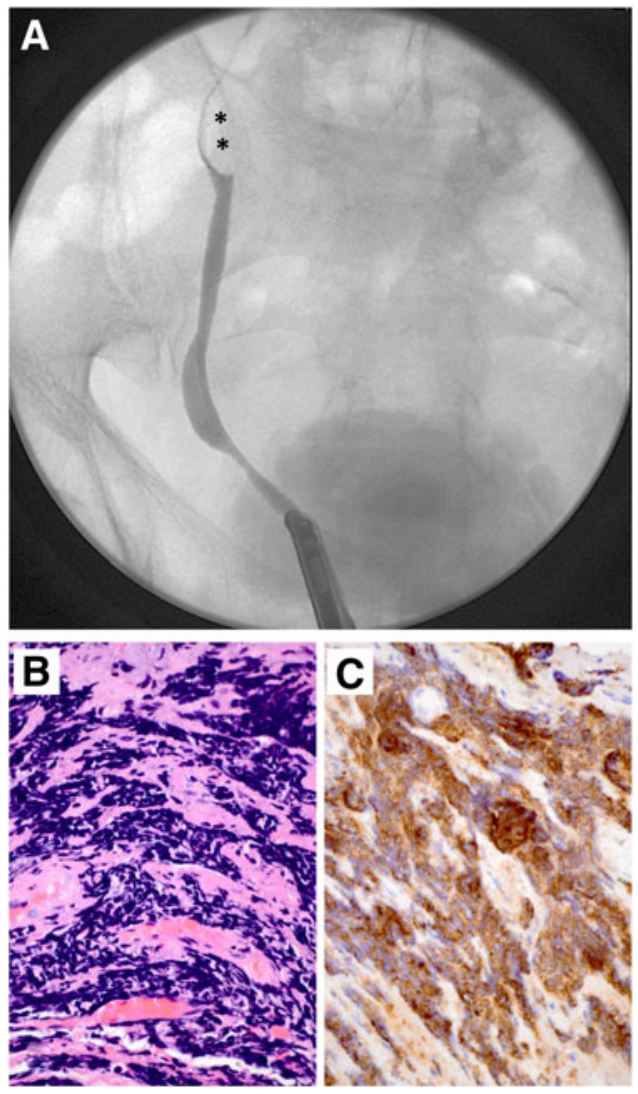

FIG. 2. Retrograde pyelogram characterized by the classic "Goblet Sign" with filling defect (asterisks) indicative of ureteral obstruction from Case 1 (A). Hematoxylin and eosinstained section revealing clusters of small palisading cells with hyperchromatic nuclei and crushed artifact $(600 \times, \mathbf{B})$. Cam 5.2 staining specific for neuroendocrine differentiation $(600 \times, \mathbf{C})$.

small left renal mass. She underwent ureteroscopy that revealed a pedunculated mass in the distal left ureter. The patient underwent laparoscopic left nephroureterectomy. Pathology revealed SCC of the distal left ureter with one para-aortic lymph node positive for metastatic SCC and positive periureteral soft tissue margins, pT4N1. She also had an incidental type I papillary renal cell carcinoma, pT1a. Immunohistochemical analysis of the ureteral tumor revealed positive staining for synaptophysin, chromogranin, and pancytokeratin. The tumor stained negative for CD45 and P63.

The patient subsequently developed pelvic lymphadenopathy on restaging imaging 5 weeks after nephroureterectomy. She underwent four courses of cisplatin and etoposide followed by consolidative external beam radiation to the pelvis. She developed metastasis to the left adrenal gland as well as recurrence in the pelvis. She developed acute respiratory failure secondary to a malignant pleural effusion. The patient was transferred to the hospice service and died 7 months after her initial diagnosis.

\section{Case 3}

A 45-year-old Caucasian female presented with abdominal pain. She was a current one pack-per-day smoker and medical history included obesity, hypertension, and previous myocardial 
infarction. Her ECOG performance status was 1 at presentation. Imaging showed a $9 \mathrm{~cm}$ right renal mass (Fig. 1C), multiple liver lesions concerning for metastases, and a right adnexal mass. She underwent percutaneous biopsy of a liver lesion that showed metastatic neuroendocrine carcinoma. She underwent open right radical nephrectomy, retroperitoneal lymph node dissection (RPLND), hepatic metastasectomy, total abdominal hysterectomy, and bilateral salpingo-oophorectomy. Pathology analysis revealed primary SCC of the right kidney with liver metastases and positive interaortocaval (2/2 positive nodes) and paracaval (2/2 positive nodes) lymph nodes. All margins of resection were negative and the final pathologic stage of the renal neuroendocrine tumor was pT3N1M1. The tumor and metastases stained positive for synaptophysin and CD56 but negative for chromogranin, CD10, CD7, and CD20. She also had a FIGO grade 1 serous tumor of the right ovary and high-grade endometrial dysplasia. Her postoperative course was complicated by a myocardial infarction and cardiac arrest necessitating cardiopulmonary resuscitation.

The patient developed bulky retroperitoneal lymphadenopathy in her initial 3-month postoperative restaging imaging and underwent repeat RPLND. Pathology analysis revealed metastatic SCC in 2/4 lymph nodes. She was subsequently lost to follow-up and re-presented 16 months after her initial diagnosis with further retroperitoneal adenopathy. Repeat RPLND showed metastatic SCC in 2/17 lymph nodes.

She was again lost to follow-up and returned 36 months after initial diagnosis with supraclavicular and retroperitoneal lymphadenopathy and hepatic and skeletal metastases. She was managed with octreotide for 2 years. Imaging revealed disease progression and she was transitioned to salvage temozolomide and capecitabine, which she tolerated for 3 months before being admitted with acute onset multisystem organ failure. She died 80 months after her initial diagnosis.

\section{Discussion and Literature Review}

The true incidence of primary upper tract SCC is difficult to define given its rarity and common pathologic misclassification. The genitourinary tract is the second-most common extrapulmonary origin of SCC to the gastrointestinal tract. ${ }^{1}$ Endoscopically, SCC of the upper and lower urinary tract is characterized by broad-based polypoid and endophytic masses that are often indistinct from papillary urothelial lesions. Two staging systems of extrapulmonary SCC exist: (1) Veterans Administration Lung Study Group system described for primary lung tumors, which assigns patients into limited (tumor confined to a single radiation therapy treatment field without nodal metastases) or extensive disease categories and (2) American Joint Committee on Cancer TNM system. ${ }^{3}$ Proposed etiologies of neuroendocrine malignancies in the urinary tract include neuroendocrine differentiation of urothelial cells or pluripotent stem cells, presence of neuroendocrine cells within the urinary tract, or trapped neural crest cells during embryology. ${ }^{3}$

SCC is histologically characterized by small, palisading cells with a high nuclear:cytoplasm ratio and high mitotic index. Immunohistochemical signatures common to these tumors include positive staining for the neuroendocrine markers CD56, synaptophysin, neuron-specific enolase, and chromogrannin $\mathrm{A}$ as well as pancytokeratin and low molecular weight cytokeratin (CAM 5.2). In a review of histology from 10 patients initially diagnosed with primary upper tract SCC, three cases were reclassified as either poorly differentiated squamous cell carcinoma or urothelial carcinoma. ${ }^{1}$ SCC commonly coexists with other primary malignancies, including urothelial carcinoma in $62 \%$ of cases. ${ }^{2}$ In addition, it is prudent to exclude metastatic pulmonary SCC to the upper tract, which has been reported. ${ }^{1}$

The median age of presentation of upper tract SCC is 66.5 years, with a male predominance $(64 \%)$. In the largest metaanalysis of upper tract SCC, $72 \%$ of patients presented with either pT3 or pT4 disease. Although only 3\% of cases reported had detectable distant metastases at the time of diagnosis, 54\% developed metastases at a median time of 13 months from diagnosis. ${ }^{2}$

Multimodal regimens have been implemented for the treatment of upper tract SCC, including surgery, radiation, and adjuvant chemotherapy with platinum-based regimens. Median survival after diagnosis ranges from 8.2 to 23 months. ${ }^{1-3}$ In the largest study of therapeutic outcomes in upper tract SCC, median survival was higher in those patients who received adjuvant chemotherapy, although this did not reach statistical significance (24 months vs 12 months; $p=0.56) .{ }^{2}$ Radiographic response to neoadjuvant cisplatin-based regimens as well as irinotecan has also been reported. ${ }^{3,4}$

The mean age of our cohort at the time of diagnosis was 67 years. Two patients presented with node positive disease at the time of surgery. Notably, patient 3 underwent extensive lymphadenectomy and hepatic metastasectomy at her index operation followed by debulking of all visible retoperitoneal disease on two occasions. She was then managed with octreotide resulting in progression-free survival for 2 years before being switched to salvage temozolomide and capecitabine. This is the first reported use of the somatostatin analogue in the management of upper tract SCC. This patient (pT3N1M1), managed with aggressive and repetitive surgical resection and multimodal therapy, experienced an 80-month survival, the longest disease-specific survival in a patient with metastatic upper tract SCC reported (previously 15 months in a patient with pT3N0M1 disease managed with nephroureterectomy). ${ }^{2}$ Conversely, patient 1 represents the first disease-specific mortality reported in a patient with upper tract SCC who received no extirpative surgery or chemotherapy. He died within 2 months of biopsy-proven diagnosis, emphasizing the aggressive natural course of this disease and further reinforcing the role of extirpative surgery.

\section{Conclusion}

Primary ureteral SCC is an exceedingly rare malignancy characterized by aggressive tumor behavior and therapeutic resistance. Previous series have observed improved diseasespecific survival in patients managed with adjuvant chemotherapy relative to surgery alone. The current series highlights the importance of combining classic radiographic and endoscopic findings with expert histopathologic evaluation to distinguish common urothelial lesions from SCC, which portends a dramatically different therapeutic regimen and prognosis. Our series emphasizes the combined role of aggressive resection of all visible disease, including lymphadenectomy, metastasectomy, and resection of recurrent, localized disease, 
with multimodal adjuvant chemoradiation to improve oncologic outcomes in this rare disease entity.

\section{Acknowledgment}

The authors thank Dr. Luis Samayoa, Professor, University of Kentucky Department of Pathology, for his pathology expertise and assistance in construction of Figure 2.

\section{Author Disclosure Statement}

Case reports are granted submission waver from the Institutional Review Board at the University of Kentucky. The authors were compliant with Health Insurance Portability and Accountability Act requirements and regulations. No competing financial interests exist.

\section{References}

1. Miller RJ, Holmäng S, Johansson SL, et al. Small cell carcinoma of the renal pelvis and ureter: Clinicopathologic and immunohistochemical features. Arch Pathol Lab Med 2011; 135:1565-1569.

2. Ouzzane A, Ghoneim TP, Udo K, et al. Small cell carcinoma of the upper urinary tract (UUT-SCC): Report of a rare entity and systematic review of the literature. Cancer Treat Rev 2011;37:366-372.

3. Ping JH, Chen ZX, Jiong Q, Han YQ, Nong X. Small cell neuroendocrine carcinoma of the ureter: A case report and literature review. Oncol Lett 2014;7:728-730.
4. Sood A, Williamson SR, Leavitt DA. Neuroendocrine tumor of the ureter: A zebra among horses. J Endourol Case Rep 2016;2:204-208.

Address correspondence to: Shubham Gupta, MD

Department of Urology

University of Kentucky College of Medicine

138 Leader Avenue

Lexington, $K Y 40506$

E-mail: shubham.gupta@uky.edu

\begin{tabular}{rl|} 
& Abbreviations Used \\
$\mathrm{CT}=$ computed tomography & ECOG $=$ Eastern Cooperative Oncology Group \\
$\mathrm{RPLND}=$ & retroperitoneal lymph node dissection \\
$\mathrm{SCC}=$ & small cell carcinoma
\end{tabular}

Cite this article as: Hensley PJ, Bhalodi AA, Gupta S (2017) Primary upper urinary tract small cell carcinoma: a case series and literature review, Journal of Endourology Case Reports 3:1, 165-168, DOI: 10.1089/ cren.2017.0103. 